\title{
Obtención de muestras de óxidos a bajo costo
}

\section{Obtaining samples of oxides at low cost}

\author{
Miryam Rincón-Joya ${ }^{1 a, 3}$, José José Barba-Ortega ${ }^{1 b}$, Elaine C. París ${ }^{2}$ \\ ${ }^{1}$ Departamento de Física, Universidad Nacional de Colombia, Bogotá, Colombia. \\ Email: ${ }^{a}$ mrinconj@unal.edu.co, ${ }^{b}$ jjbarbao@unal.edu.co \\ ${ }^{2}$ Embrapa Instrumentação, Centro de pesquisa Agropecuaria, Rua XV de Novembro 1452, 13560-970, São Carlos SP, Brasil. \\ Email: elaine.paris@embrapa.br \\ ${ }^{3}$ Foundation of Researchers in Science and Technology of Materials, Bucaramanga, Colombia
}

Recibido: 22 octubre, 2018. Aceptado: 11 enero, 2019. Versión final: 21 marzo, 2019.

\section{RESUMEN}

En este trabajo se presenta un estudio comparativo de tres métodos en la obtención de muestras a bajo costo. Las muestras obtenidas son nanopaticulas de óxido de Hierro, óxido de Cobre y nanotubos de Titanio. Las dos primeras fueron obtenidas por el método Sol-Gel, precursores poliméricos y la tercera muestra por el método de anodización química. Las muestras fueron caracterizadas por métodos ópticos como Raman, Rayos X y microscopía electrónica de barrido. Los métodos usados para la obtención de las muestras son métodos químicos de los cuales se pueden obtener excelentes muestras a un costo bajo y con el uso de laboratórios simples, sobre todo en Colombia donde las inversiones en laboratorios de investigación es baja hasta el momento.

PALABRAS CLAVE: Sol-Gel; Métodos Ópticos; Nanomateriales.

\begin{abstract}
In this work we present a comparative study of three methods in obtaining samples at low cost. The samples obtained are nanopaticules of Iron oxide, copper oxide and Titanium nanotubes. The first two were obtained by the Sol-Gel, polymeric precursors and the third sample by the chemical anodization method. The samples were characterized by optical methods such as Raman, X-rays and scanning electron microscopy. The methods used to obtain the samples are chemical methods from which excellent samples can be obtained at a low cost and with the use of simple laboratories, especially in Colombia where the investment in research laboratories is low so far.
\end{abstract}

KEYWORDS: Sol-Gel; Optical Methods; Nanomaterials.

\section{INTRODUCCIÓN}

En la literatura existen muchos métodos de síntesis de materiales y también con diferentes dificultades en la obtención de varios tipos de nanopartículas [1], [2], [3], [4], [5], [6], [7]. Sin embargo, no siempre es posible tener acceso a todos los equipos de laboratórios complejos. Por eso, desde nuestro punto de vista, se hace necesario cada vez desarrollar más métodos de síntesis con óptimos re- sultados y a un precio razonable en la obtención de nuevos materiales. En las diferentes revistas de investigación se pueden encontrar varios artículos con diferentes técnicas tanto físicas, químicas, Biológicas y Electroquímica en la obtención de materiales [1], [2], [4], [5]. No obstante, en el momento de elegir el método de síntesis de los materiales también hay que tener en cuenta que resultados se quieren obtener de las muestras. A modo de 
ejemplo aquí, presentaremos tres muestras obtenidas por diferentes métodos de síntesis. Anodización, Precursores poliméricos y Sol-Gel. El óxido de cobre $(\mathrm{CuO})$ es uno de los semiconductores de tipo $p$ con una banda prohibida indirecta, este material tiene una atención considerable debido a sus excelentes propiedades ópticas, eléctricas, físicas y magnéticas. $\mathrm{El} \mathrm{CuO}$ se usa ampliamente en diversas aplicaciones, como catálisis, conversión de energía solar, sensor de gas y emisión de campo [8], [9]. Los nanotubos de dióxido de titanio $(N T T)$ han atraído una amplia atención y se han aplicado extensivamente en el campo de la biomedicina, debido a su gran área de superficie específica, buena resistencia a la corrosión, excelente biocompatibilidad y bioactividad mejorada. En particular, la aplicación de NTT en biosensores ha atraído mucha atención debido a su capacidad para el diagnóstico rápido de enfermedades [10]. El óxido de hierro, $\mathrm{Fe}_{2} \mathrm{O}_{3}$ o magnetita, es uno de los principales óxidos del hierro estudiados. Su importancia radica en sus propiedades químicas y físicas. En primer lugar, el color rojo vivo que manifiesta le confiere utilidad como pigmento, puede funcionar también como un aislante eléctrico, para el tratamiento de aguas residuales. En la literatura se encuentran varias publicaciones en el estudio de este compuesto [11]. Con base en este contexto, este trabajo tiene por objetivo mostrar tres diferentes métodos de síntesis de estos materiales, estos métodos son de fácil acceso y de bajo costo. Además mostraremos los modos vibracionales de los óxidos de hierro, titanio y cobre. En este trabajo también se observó la morfología de las muestras obtenidas por diferentes rutas de síntesis. Además comparamos las síntesis del óxido de cobre, óxido de hierro y óxido de Titanio entre ellas. Entre los métodos de síntesis químicos reportados en la literatura para la producción de óxidos metálicos, constituidos por partculas nanométricas, el método Pechini, también conocido como método de los precursores poliméricos, se ha visto como un método prometedor para la obtención de polvos de cerámica, ya que este método permite la utilización de diferentes rangos de temperatura y diferentes proporciones de cationes metálicos, lo que es importante para una buena homogeneidad, alta cristalinidad y con dimensiones nanométricas [10]. La descripción de los materiales usados en el método de pechini para la obtención de las nanoparticulas de hierro se encuentran en la referencia [11]. El método Sol-Gel es un proceso químico en fase húmeda ampliamente utilizado en la ciencia de los materiales. El proceso Sol-Gel consta de varios pasos: mezclado, gelificación, envejecimiento, secado y sinterizado [12]. En la síntesis Sol-Gel partiendo de los precursores moleculares como alcóxidos metálicos o sales inorgánicas, su resultado final será un esqueleto del óxido mediante reacciones de hidrolisis y polimerización a baja temperatura, permitiendo las fases metaestables del óxido [12]. Una característica particular del proceso solgel es la posibilidad de controlar el proceso de síntesis desde el precursor molecular al resultado final [12]. Entre las ventajas del método está: alta homogeneidad de las disoluciones, obtención de materiales en forme de fibras, partículas , esferas etc, mnima contaminación del aire, área superficial alta. Los problemas más comunes en este proceso son: El costo de los materiales de partida, presencia de poros residuales, larga duración del proceso. Los materiales usados para la obtención del óxido estn descritos en otra publicación [13]. En la literatura existen muchas publicaciones del procesos de síntesis de nanotubos por anodización [14]. Este proceso electroquímico modifica la morfología superficial, de un sustrato a partir de la oxidación disolución de una capa de óxido. Para la obtención de los nanotubos es necesario entender el mecanismo de formación. Teniendo en cuenta, que primero, se forma el óxido en la superficie de la lámina; aquí se tiene una caída de corriente. En la siguiente etapa, la capa de óxido formada presenta una alta resistencia afectando la corriente hasta alcanzar valores mnimos. Es decir, en la superficie se forma un óxido metal. En la etapa III, hay formación de nanoporos en la superficie. Por último, empieza la formación de los nanotubos. Formados por la competencia entre la oxidación y disolución. La morfología de los nanoporos y nanotubos están determinados por los componentes químicos, tiempo, voltaje y distancia del ánodo y el cátodo. La ventaja de este método es también el bajo costo, los implementos usados son muy simples. La dificultad está en encontrar las mejores variables, para obtener los mejores resultados en la obtención de las nonoestructuras [14]. Los productos obtenidos fueron sometidos a análisis de caracterización de rayos X para la identificación de las fases formadas en la estructura de las muestras. Espectroscopia Raman con el objetivo de obtener los modos vibracionales y microscopia electrónica de barrido $(M E B)$ en la obtención de la morfología de las mismas [15].

\section{RESULTADOS}

En la figura 1(a), se observa los resultados de las medidas realizadas sobre una muestra de $\mathrm{TiO}_{2}$, obtenida por el método de anodización. La muestra fue sometida a 20 $\mathrm{V}$, durante 60 minutos. Luego, la muestra fue sometida a una temperatura de calcinación de $450^{\circ} \mathrm{C}$. En esta medida de Microscopía Electrónica de Barrido con cañón de emisión de campo $(M E B-F E G)$ del material nanoestructurado. En esta muestra se observa que los nanotubos de $\mathrm{TiO}_{2}$, tiene más de $300 \mathrm{~nm}$ de largo y menos de $50 \mathrm{~nm}$ de diámetro. Como se puede apreciar la morfología de la muestra, creció homogeneamente. La espectroscopia 

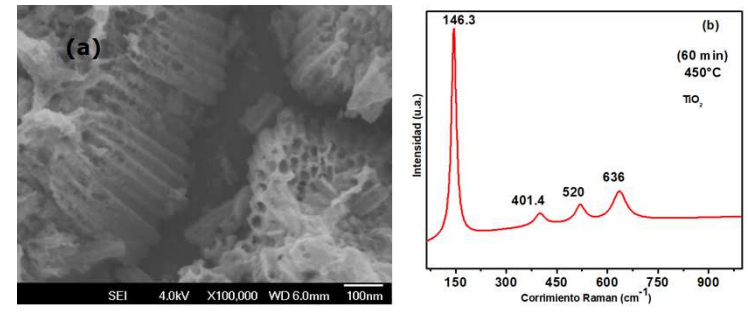

Figura 1: (a) Morfología de la muestra de $\mathrm{TiO}_{2}$ obtenida a $450^{\circ} \mathrm{C}$ en un tiempo de $60 \mathrm{~min}$ y un potencial de $20 \mathrm{~V}$ (MEB-FEG). (b) Espectro Raman de la muestra (a), excitada con un laser de 632nm.

Raman es una herramienta poderosa y prometedora en el estudio de las microestructuras de los nanotubos de $\mathrm{TiO}_{2}$. Todos los espectros Raman medidos a temperatura ambiente en la muetras de $\mathrm{TiO}_{2}$ son asignados a la fase anatasa del dióxido de Titanio. Los modos vibracionales de la fase anatasa son: $144\left(\mathrm{E}_{g}\right), 197\left(\mathrm{E}_{g}\right), 399\left(\mathrm{~B}_{1 g}\right), 513$ $\left(\mathrm{A}_{1 g}\right), 519\left(\mathrm{~B}_{1 g}\right)$ y $639\left(\mathrm{E}_{g}\right) \mathrm{cm}^{-1}$ [16]. De los seis modos Raman mencionados en la literatura del material en la figura 1 (b), solamente se alcanza a observar cuatro, posiblemente los otros dos están enmascarados en los modos presentes, ya que estos se observan muy intensos. En
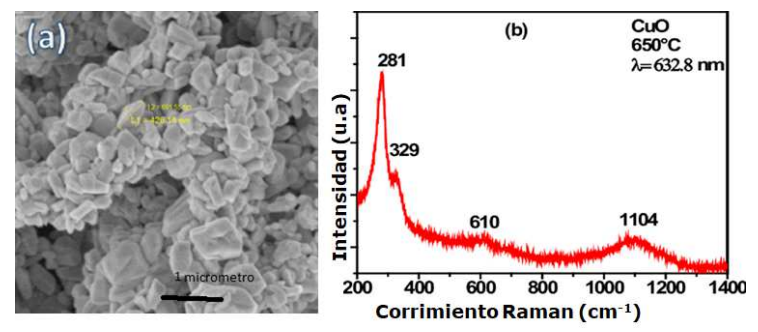

Figura 2: (a) Microscopía electrónica de barrido de la muestra de $C u O$, calcinada después del proceso a $650^{\circ} \mathrm{C}$. (b). Espectro Raman de $\mathrm{CuO}$, excitada con un laser de $632.8 \mathrm{~nm}$.

el óxido de cobre, los patrones de DRX mostraron que los nanomateriales sintetizados estaban en una estructura cristalina monoclnica [13]. En la figura 2(a), se observa una muestra con obtenida por el método sol-gel. La muestra fue calcinada a $650^{\circ} \mathrm{C}$, sin embargo como se observa en otro trabajo [13], la estructura realmente se puede obtener a $450^{\circ} \mathrm{C}$, con un tamaño de partícula menor. Como se puede apreciar en la medida MEB, las nanopartículas crecieron de forma regular, sin embargo al sometarla a altas temperaturas de calcinación, las nanopartículas de las muestras crecieron bastante, pero menor a un micrometro. En la figura 2(b), se observan los modos vibracionales del $\mathrm{CuO}$. Como se aprecia, la estructura cristalina del material fue obtenida. Hay nueve modos de fonones ópticos de centro de zona con simetrías $4 \mathrm{Au}+$ $5 \mathrm{Bu}+\mathrm{Ag}+2 \mathrm{Bg}$; solo tres modos $\mathrm{Ag}+2 \mathrm{Bg}$ son Raman activos. La medida de espectrocopía Raman, se llevó a cabo a temperatura ambiente, los modos vibracionales corresponden a la estructura monoclinica del $\mathrm{CuO}$. En la
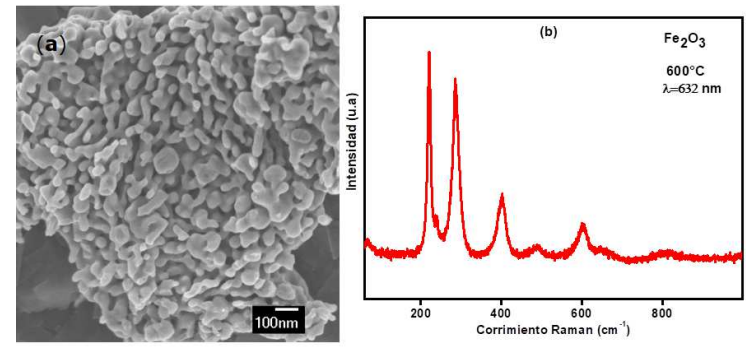

Figura 3: (a) Microscopía electrónica de barrido de la muestra de $\mathrm{Fe}_{2} \mathrm{O}_{3}$, calcinada después del proceso a $600^{\circ} \mathrm{C}$. (b). Espectro Raman de $\mathrm{Fe}_{2} \mathrm{O}_{3}$, excitada con un laser de $632 \mathrm{~nm}$.

figura 3, presentamos la muestra de óxido de hierro hematita, preparada por el método de precursores poliméricos (método de Pechini). El material fue precalcinado a $300^{0} \mathrm{C}$ por 2 horas y luego fue sometido a calcinación a una temperatura de $600^{\circ} \mathrm{C}$. En la figura 3 (a), se observa la morfología del material. Las nanopartculas obtenidas son menores a 100nm y presentan una homogeneidad en su formación. Esto indica, que la temperatura de calcinación aplicada es la adecuada para la obtención de la muestra hematita con nanopartículas uniformes. Los espectros Raman (figura 3(b), confirman la presencia de la fase pura de $\mathrm{Fe}_{2} \mathrm{O}_{3}$ que es soportada por los datos de $D R X$ [11]. Los picos a 222 y $495 \mathrm{~cm}^{-1}$ corresponde a $\mathrm{A}_{1 g}$ y los picos a 244, 290, 406 y $608 \mathrm{~cm}^{-1}$ corresponde a $\mathrm{E} g$ de los modos vibracionales de $a-\mathrm{Fe}_{2} \mathrm{O}_{3}$. Con este espectro Raman, se confirma la obtención de la fase pura por este método de síntesis, sin picos adicionales [17]. El modo más intenso que se observó en la medida Raman fue en aproxidamente $222 \mathrm{~cm}^{-1}$ del $\mathrm{A}_{1 g}$, luego, en intensidad sigue el modo Eg en $290 \mathrm{~cm}^{-1}$. Como se puede apreciar, las muestras obtenidas por este método de síntesis, son de muy buena cristalinidad. En la figura 4, se muestra un esquema general de los tres métodos de sístesis presentados en este trabajo. Uno de estos métodos de síntesis de polvos y pelculas cerámicas, que se viene popularizando es el método de los precursores poliméricos, derivado de la patentede Pechini en 1967. El método se basa en la capacidad de los ácidos orgánicos hidroxicarboxílicos formar quelatos con varios cationes. Cuando un alcohol polihidroxi se añade a este quelato, bajo calentamiento y agitación constante. A continuación, con el calentamiento y eliminación del exceso de solvente, una resina altamente viscosa se forma. Esta resina se calcina, 


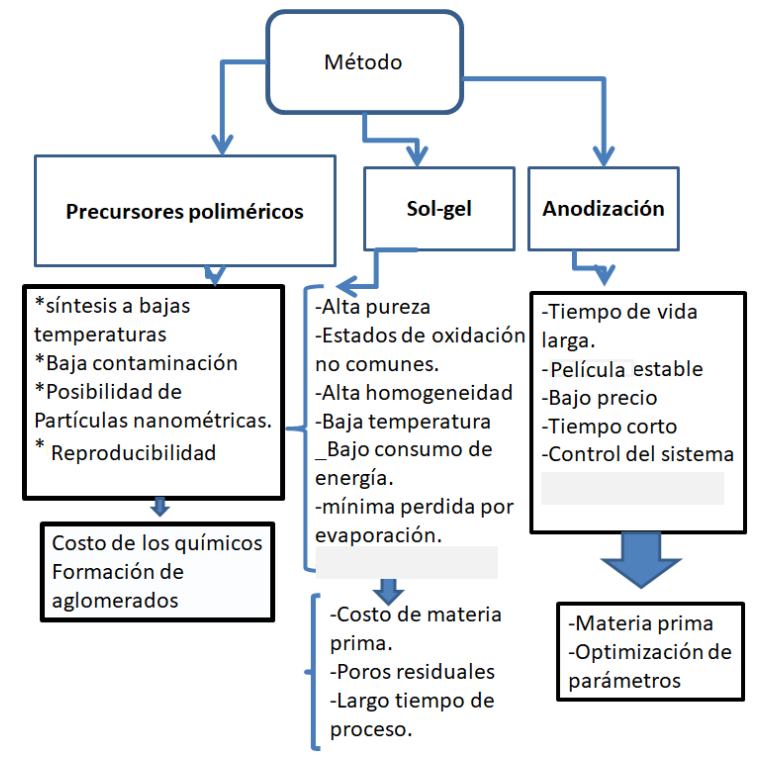

Figura 4: Flujograma de las ventajas y desventajas de los métodos de síntesis de materiales, precursores poliméricos, Sol-Gel y anodización.

generalmente a $300^{\circ} \mathrm{C}$. Luego, se realiza el tratamiento térmico a diferentes temperaturas [18]. Generalmente, el ácido cítrico es usado como agente quelante y el etilenglicol como agente polimerizante. La anodización, es una reacción de oxidación,-disolución electroquímica proceso de síntesis sencillo, con un buen control morfológico. El proceso se utiliza para modificar la morfología superficial, de un sustrato a partir de la oxidacióndisolución de una capa de óxido sobre la muestra de interés. Bósicamente, consiste en dos eléctrodos, uno como ánodo y otro como cátodo. El proceso de síntesis se realiza en una celda electroquímica [19]. El proceso Sol-Gel, en la preparación de polvos, monolitos y filmes finos es basado en la reacción de hidrolisis polimerización de precursores alcóxidos. El proceso envuelve el uso de líquidos de baja viscosidad. El Sol es descrito, como una suspensión de partículas coloidales, en cuanto al Gel se refiriere a una masa semi-rígida, se forma cuando las partículas coloidales están unidas en forma de red. La hidrolisis es la principal reacción química que conduce a la transformación de los precursores, esto es, los óxidos. La condensación es responsable por la polimerización de los precursores [20].

\section{CONCLUSIONES}

Con base en los resultados obtenidos en este trabajo, podemos concluir que las medidad de espectroscopia Raman sugieren que las muestras obtenidas son fases puras, sin adición de otros modos vibracioanles en los tres procesos de síntesis y para los tres óxidos. Los métodos Pechini, Sol-Gel y anodización utilizados en este trabajo se mostraron eficientes para la obtención de la fases cristalinas de cada una de las muestras. Sin embargo, para el óxido de cobre, es posible que aun se pueda mejorar la ruta con otros parámetros con el mismo método en el proceso. En el caso de la anodización para los nanotubos de $\mathrm{TiO}_{2}$, se mostró que los parámetros usados en el proceso son los adecuados. En las muetras de $\mathrm{Fe}_{2} \mathrm{O}_{3}$, la morfología y la fase es pura.

\section{REFERENCIAS}

[1] M. A. Ribeiro, L. S. Neiva, D. F. Maia, J. B. L. de Oliveira, L. Gama, "Síntese do $\mathrm{TiO}_{2}$ dopado com $\mathrm{Zr}$ por meio do método Pechini: Avaliação dos efeitos da temperatura de calcinação", Rev. Eletr. Mat. e Proc., vol. 7, pp.111-116, 2012.

[2] O. F . Lopes, V. R. Mendonça, F. B. F. Silva, E. C. Paris, C. Ribeiro, "Óxidos de nióbio: uma visão sobre a síntese do $\mathrm{Nb}_{2} \mathrm{O}_{5} \mathrm{e}$ sua aplicação em fotocatálise heterogénea", Quim. Nov., vol. 38, pp. 106-117, 2015.

[3] A. M. Raba, J. Bautista-Ruíz, M. R. Joya, "Synthesis and structural properties of niobium pentoxide powders: A comparative study of the growth process", Mat. Res., vol. 19, pp. 1381-1387, 2016.

[4] M. R. Joya, J Barón-Jaimez, J. Barba-Ortega, "The technique of Raman spectroscopy in the characterization physical, chemical and structural ferroelectric of the materials", J. Phys.: Conf. Ser., vol. 466, p. 012007, 2013

[5] M. R. Joya, J. H. Bautista Ruíz, A. M. Raba. "Quicklime as an alternative in the photodegradation of contaminants", J. Phys.: Conf. Ser., vol. 687, p. 012044, 2016.

[6] S. Rozo-Rincón, J. Gelvez-Díaz, J. Sanchez-Molina, J. BautistaRuíz, Çaracterización de cerámicos inmersos en soluciones del sistema $\mathrm{Sio}_{2}$, $\mathrm{tio}_{2}, \mathrm{zro}_{2}$ sintetizadas por el método sol-gel", Rev. UIS Ing., vol. 16, no. 2, pp. 51-59, 2017.

[7] Y. M. Franco, C. Ortiz, J. E. R. Paéz, J. H. B, Ruíz, .obtención y caracterización de recubrimientos de $\mathrm{TiO}_{2}$ por el método de complejo polimerizable (PECHINI)", Respuestas, vol. 15, no. 1, pp. 25-32, 2010.

[8] K. Phiwdang, S. Suphankij, W. Mekprasart, W. Pecharapa, "Synthesis of $\mathrm{CuO}$ nanoparticles by precipitation method using different precursors", Energy Proced, vol. 34, pp. 740-745, 2013.

[9] A. F. Zedan, A. T. Mohamed, M. S. E.Shall, S. Y. A.Qaradawia A. S. A.Jabera, "Tailoring the reducibility and catalytic activity of $\mathrm{CuO}$ nanoparticles for low temperature $C O$ oxidation", $R S C$ Adv., vol. 35, 2018.

[10] M. A. Ribeiro, L. S. Neiva, D. F. Maia, J. B. L. de Oliveira, L. Gama, "Sintese do $\mathrm{TiO}_{2}$ dopado com $\mathrm{Zr}$ por meio do mtodo Pechini: Avaliação dos efeitos da temperatura de calcinação", Rev. Eletr. Mat e Proc., vol. 7, no. 2, pp. 111-116, 2012.

[11] M. R Joya, J Barón-Jaimez, J. Barba-Ortega, "Preparation and characterization of $\mathrm{Fe}_{2} \mathrm{O}_{3}$ nanoparticles", J.Phys.: Conf. Ser, vol. 466, p. 012004, 2013.

[12] M. Rojas, "Diseño y síntesis de materiales a medida mediante el método sol-gel", MSc Thesis, Universidad Nacional de Educación a Distancia, Madrid, España, 2012. 
[13] M. R. Joya , J. Barba-Ortega, A. Raba, "Vibrational Raman modes and particle size analysis of cupric oxide with calcination temperature", J. Pure. Appl. Phys., accepted, 2018.

[14] H. M. Mateus, J. Barba-Ortega, M. R. Joya, Çomparison of the Growth of Nanotubes in Different Solutions", J. Inor. Organ. Polym. Mat., vol. 28, no. 3, pp. 612-623, 2018.

[15] Ely Dannier Valbuena-Nio, Miryam Rincón-Joya, and Jos Jos Barba-Ortega, "Study of structural modifications in a ferroelectric material by means of two optical methods", Iteckne, vol. 14, no. 2, pp. 105-109, 2017.

[16] M. J. Scepanovi, M. G.Brojcin, Z. D. D.Mitrovi, Z. V. Popovic, Çharacterization of Anatase $\mathrm{TiO}_{2}$ Nanopowder by VariableTemperature Raman Spectroscopy", vol. 41, pp. 67-73, 2009.

[17] A. Lassoued, B. Dkhil, A. Gadri, S. Ammar, Çontrol of the shape and size of iron oxide $\left(\alpha-\mathrm{Fe}_{2} \mathrm{O}_{3}\right)$ nanoparticles synthesized through the chemical precipitation Method", Resul. Phys., vol. 7, pp. 3007-3015, 2017.

[18] G. L. G. T. Botelho,J. A. N. de Oliveira, M. R. C. Santos,M. Zampieri, E. Longo, "Vantagens na utilização do método dos precursores poliméricos na síntese do $\mathrm{Mg}_{2} \mathrm{SnO}_{4}$ ", Soc. Bras. de Qum., p. 33, 2010.

[19] A. A. Hernández "Síntesis y caracterización de nanotubos de dióxido de Titanio", MSc Thesis, Instituto Nacional de Astrofísica, Óptica y Electrónica, Mexico, 2016.

[20] Z. M. Lima, "Nova rota de síntese de nanoparticulas $\mathrm{NiMn}_{2} \mathrm{O}_{4}$ usando o método sol-gel proteic", MSc Thesis, Universidade Federal do Ceará, Brasil, 2011. 